\title{
TITLE:
}

\section{Photocurable electrolyte based on sulfonated poly(ether ether ketone)}

AUTHOR(S):

Nagao, Yuki; Iwadera, Tomoaki; Sata, Noriko; Iguchi, Fumitada; Yugami, Hiroo

\section{CITATION:}

Nagao, Yuki ... [et al]. Photocurable electrolyte based on sulfonated poly(ether ether ketone). Solid State Ionics 2011, 204-205: 35-40

\section{ISSUE DATE:}

2011-12-12

URL:

http://hdl.handle.net/2433/152423

\section{RIGHT:}

(C) 2011 Elsevier B.V.; This is not the published version. Please cite only the published version.; この論文は出版社版でありません。引用の際に は出版社版をご確認ご利用ください。 


\title{
Photocurable Electrolyte Based on Sulfonated Poly(ether ether ketone)
}

Yuki Nagao ${ }^{\mathrm{a}, \mathrm{b}}$, Tomoaki $\operatorname{Iwadera}^{\mathrm{c}}$, Noriko Sata $^{\mathrm{c}}$, Fumitada Iguchi $^{\mathrm{c}}$, Hiroo Yugami $^{\mathrm{c}}$

${ }^{a}$ Division of Chemistry, Graduate School of Science, Kyoto University, Kitashirakawa

Oiwake-cho, Sakyo-ku, Kyoto 606-8502, Japan, ${ }^{\mathrm{b}}$ PRESTO, Japan Science and

Technology Agency (JST), 4-1-8 Honcho Kawaguchi, Saitama 332-0012, Japan, and

${ }^{\mathrm{c}}$ Department of Mechanical Systems and Design, Graduate School of Engineering,

Tohoku University, 6-6-01 Aramaki Aoba, Aoba-ku, Sendai 980-8579, Japan

Corresponding author: ynagao@kuchem.kyoto-u.ac.jp

\begin{abstract}
Photocurable electrolytes were prepared by mixing proton conductive polymer sulfonated poly(ether ether ketone) (SPEEK), cross-linking monomer divinyl sulfone (DVS), photoinitiator phenylbis(2,4,6-trimethylbenzoyl)phosphineoxide (PI), dimethylacetamide (DMA), and ethanol. To investigate the proton transport property, a series of photocurable electrolytes with various PI content ratios were prepared to form photocured membranes. Water uptake, ion exchange capacity (IEC), proton conductivity,
\end{abstract}


and water-resistance of the photocured membranes were investigated. Water-resistant photocured membrane is easy to prepare, which gives relatively high proton conductivity $\left(28 \mathrm{mS} \mathrm{cm}^{-1}\right.$ under $70{ }^{\circ} \mathrm{C}$ and relative humidity of $\left.90 \%\right)$. Patterning of micro-structure on the photocured membrane was also demonstrated by using the property of the photocurable electrolytes.

\section{Keywords}

Proton conduction, Photocuring, Semi-interpenetrating network, photoinitiator content 


\section{Introduction}

The polymer electrolyte membrane (PEM) fuel cells are one of the ideal power sources for portable electronic devices with high power-to-weight ratios and fast start-up performance. Nafion is employed as pre-cast membranes, and is the most widely studied PEM because it exhibits high conductivity, good mechanical stability, and is commercially available. In general, preformed membranes and catalyzed electrodes are separately prepared in solid state. A high-pressure press at a softening temperature of the membrane is used to connect two catalyzed electrodes on both sides of the membrane. This method is called hot-pressing. There are few reports that have utilized liquid phase of the electrolytes to connect the membrane with the catalyzed electrodes without hot-pressing and the hot-pressing process is essential to prepare membrane-electrode assemblies for solid-state membranes.

Catalytic reactions mainly occur at triple phase boundary, which consist of the catalyst, proton conducting electrolyte, and gaseous reactant. Proton-conductive ionomers such as Nafion solution are used to fabricate suitable triple phase boundary. A liquid electrolyte can be one of the useful ionomers not only to fabricate the membrane but also to work as binder to form triple phase boundary because of their excellent properties of proton conductivity and penetration to the catalytic layer in the electrode. 
We focused on photocurable liquid electrolytes, which may enable the formation of membrane-electrode assemblies without hot-pressing. Holdcroft and co-workers [1] proposed the concept of making PEMs from curable liquid precursors and pointed out that such a liquid-to-PEM approach may enable the formation of PEMs conformable by injection molding, formed as microchannels and unique shapes [2], or strongly adhering to the catalyst layer without hot pressing [3]. Therefore, the liquid-to-PEM approach does not need any high-pressure pressing at high temperature.

A photocurable technique may also contribute to the preparation of a water-resistant membrane. A mixture of two or more preformed polymer networks does not have an interpenetrating network [4]. If the preformed polymer is soluble in water, the preformed polymer in the mixture would be soluble in water. However, the preformed polymer in the semi-interpenetrating network might not dissolve due to the interpenetrating architecture.

In this study, we prepared the photocurable electrolytes by mixing proton conductive polymer, cross-linking monomer, photoinitiator (PI), and solvent. As the proton conductive polymer, sulfonated poly(ether ether ketone) (SPEEK) was chosen because of its solubility in a variety of solvents and its high proton conductivity. Divinyl sulfone (DVS) was chosen as the cross-linking monomer because of its cross-linking ability and 
solubility in a variety of solvents. Phenylbis(2,4,6-trimethylbenzoyl)phosphineoxide was chosen as the PI because of its solubility in a variety of solvents. The role of dimethylacetamide (DMA) and ethanol was to control the viscosity of the liquid solution. It is well known that SPEEK with a high sulfonation degree (SD) is highly proton-conductive polymer, but it dissolves in water. It is essential to synthesize water-resistant membranes with SPEEK, and the photocurable technique is one of the key methods to solve this problem.

A few groups have investigated the characteristic changes that derived from the content ratio of the proton conducting polymer or monomer [1,5]. Holdcroft and co-workers investigated the relationship between the proton conductivity and SPEEK content [1]. However, the effect of the PI content ratio on the proton conductivity has not been reported and unfamiliar. We found that the PI content ratio was one of the important parameters to the property of the proton-conductive membrane. In this study, we prepared a series of photocurable electrolytes which had various PI and solvent content ratios in order to investigate the effects of PI content and solvent on the proton conductivity. Water uptake, ion Exchange Capacity (IEC), proton conductivity, and water-resistance of the prepared membranes were investigated. 


\section{Experimental}

\subsection{Materials}

Figure 1 shows the chemical structures of the materials used in this work. Poly(ether ether ketone) (PEEK) was purchased from Sigma-Aldrich Japan K.K. in powder form, which had a molecular weight Mw 20,800, and a density of $1.32 \mathrm{~g} \mathrm{~cm}^{-3}$. Sulfuric acid (95 wt\%), dimethylacetamide (DMA) and ethanol were purchased from Wako Pure Chemical Industries Ltd. The photoinitiator, phenylbis(2,4,6-trimethylbenzoyl)phosphineoxide, was purchased from Sigma-Aldrich Japan K. K. Divinyl sulfone (DVS) was purchased from Tokyo Chemical Industry Co., Ltd. All of the materials were used as received.

\subsection{SPEEK preparation}

$9 \mathrm{~g}$ of PEEK was dissolved in $300 \mathrm{~mL}$ of sulfuric acid at room temperature via mechanical agitation in a three-neck flask under an Ar atmosphere [6-8]. After complete dissolution of the PEEK, the polymer solution was heated to $55^{\circ} \mathrm{C}$ for sulfonation for 6 hrs. The sulfonated SPEEK solution was quenched in a significant excess of deionized ice water to recover the modified polymer. Obtained polymer was washed repeatedly 
with deionized ice water until the $\mathrm{pH}$ value of the washing water was greater than 5 .

The recovered SPEEK was dried in a vacuum. Characterization of the obtained SPEEK was carried out by using ${ }^{1} \mathrm{H}$ NMR with DMSO-d6 including $0.05 \%$ TMS.

Figure 2 shows the chemical shift assignments of the ${ }^{1} \mathrm{H}$ NMR spectrum of the synthesized SPEEK. The SD of SPEEK was quantitatively determined by evaluating the intensities or the peak areas corresponding to all the protons as indicated in Fig. 2, based on the following equation [9].

$n /(12-2 n)=A_{\mathrm{HE}} / \sum A_{\mathrm{HABCDF}} \quad(0 \leq n \leq 1)$,

$S D=n \times 100 \%$

where $A_{\mathrm{HE}}$ is the area of the signal attributed to $\mathrm{H}_{\mathrm{E}}$, and $\sum A_{\mathrm{HABCDF}}$ is the area of the signals of other aromatic protons. The SD was determined to be $80 \%$ by this equation.

\subsection{Preparation of the photocurable electrolyte}

Liquid photocurable electrolytes (S1-S5) with different photoinitiator (PI) and solvent contents were prepared according to the compositions shown in Table I. The appropriate quantities of DVS, PI, and solvent were added into a vial. After complete dissolving of the photoinitiator, appropriate quantities of SPEEK were added and the mixture was kept in the dark at room temperature for 2 days until SPEEK appeared to dissolve. Then, 
the mixture was heated at $80{ }^{\circ} \mathrm{C}$ for 1 day in order to ensure dissolution of SPEEK.

2.4 Preparation of the photocured membranes

Synthesized liquid photocurable electrolytes were spread with a casting knife onto glass slides with $\mathrm{Cu}$ wire spacers $(0.3 \mathrm{~mm}$ in diameter). The photocured membranes ( $\mathrm{S} 1$ - S5 membranes) were obtained by the light irradiation using a xenon short arc lamp (Ushio Inc. DSB-75A, XS 151A, and UXL-75D-O) until no more change in mass of the cured membrane was observed. The intensity of light irradiation was $3 \mathrm{~mW} \mathrm{~cm}^{-2}$, which was measured by ADCMT 8230 optical power meter with an 82312 Si photo-diode sensor (detectable wavelength: $390-450 \mathrm{~nm}$, calibrated wavelength: $405 \mathrm{~nm}$ ). After photocuring, the membrane was heated at $70{ }^{\circ} \mathrm{C}$ over $7 \mathrm{hrs}$ to remove the solvent DMA and/or ethanol completely.

2.5 Water uptake and ion exchange capacity measurements

Equivalent weight (EW) and IEC values of synthesized photocured membranes were determined by titration of the sulfonic acid groups. In the titration measurement, a piece of membrane (typically $0.02-0.06 \mathrm{~g}$ ) in acid form was soaked in $4.5 \mathrm{M} \mathrm{NaCl}$ solution for 1 day, and the resulting solution was then titrated with 5 mmol $\mathrm{L}^{-1} \mathrm{NaOH}$ solution 
using phenolphthalein as the indicator. The titrated membrane was in the salt form and the weight of a wet membrane was measured. The membrane was dried in a vacuum at $80{ }^{\circ} \mathrm{C}$ over $6 \mathrm{hrs}$, then the accurate membrane weight in the dried condition was measured as a dried membrane. The water uptake is calculated by the difference of the weight between dried membrane and wet membrane with following equation:

Water uptake $=[($ weight of wet membrane $/$ weight of dried membrane $)-1] \times 100$. (3)

The EW and IEC of each membrane were obtained as follows:

$\mathrm{EW}\left(\mathrm{H}^{+}\right)=\left[\right.$weight of dried membrane $\left./\left(V_{\mathrm{NaOH}} \times[\mathrm{NaOH}]\right)\right]-22$,

$\mathrm{IEC}=1000 / \mathrm{EW}$,

where $V_{\mathrm{NaOH}}$ is the volume of the $\mathrm{NaOH}$ solutions required for the titration (L).

\subsection{Proton Conductivity}

Proton conductivity $(\sigma)$ of the photocured membrane was measured by a two-probe AC impedance method over a frequency range of $1 \mathrm{~Hz}-10 \mathrm{MHz}$. Impedance spectra were recorded using a Solartron 1260 Impedance / Gain-phase analyzer and a Solartron 1296 Dielectric Interface. The conductivity cell was designed to observe the impedance response of the membrane dominantly, as demonstrated by Zawodzinski and co-workers [10]. The "window" structure of the cell allowed the membrane to equilibrate in-situ 
with the atmosphere around the membrane. The temperature dependence of the proton conductivity was investigated in the temperature range of $70-25{ }^{\circ} \mathrm{C}$ at the relative humidity $(\mathrm{RH})$ of $90 \%$. The $\mathrm{RH}$ dependence of the proton conductivity was investigated in the RH range of $90-50 \%$ at $25{ }^{\circ} \mathrm{C}$. The samples were kept in each condition for at least $1 \mathrm{~h}$ before performing any measurements. Conductivity measurements were repeated at each condition until no more changes were observed.

2.7 Preparation of the patterned structure on the surface of the membrane

To demonstrate a patterned membrane, a patterned structure on Si wafer as a mold was fabricated by the conventional techniques of micro electro mechanical systems (MEMS). To prepare the patterned membrane, the liquid photocurable electrolyte was transferred onto the patterned mold of $\mathrm{Si}$ wafer and then light curing to the liquid photocurable electrolyte was carried out for $1 \mathrm{~h}$. Before photocuring, air bubbles in the liquid were carefully removed. After photocuring, the patterned membrane was peeled off from the patterned mold of Si wafer.

\section{Results and Discussion}

3.1 Preparation of the photocured membrane 
Figures 3 show the ${ }^{1} \mathrm{H}$ NMR spectra of S2 and S3 membranes before and after heating. The intensity of three single signals $(1.96,2.79$, and $2.95 \mathrm{ppm})$ derived from the protons of DMA weakened remarkably after heating the membranes. These facts indicate that considerable amount of DMA was left in as-synthesized membranes and that the solvent could be removed by the heat treatment. It is suggested that the membranes could have vacancies upon the removal of the solvent, such as DMA, as shown in Figs. 4(a) and (b).

The mechanical properties of the photocured membranes greatly depended on the PI content. Photocured S1 membrane was obtained by curing in 2 hrs. It was very brittle and broke easily. In contrast, the as-prepared S2 and S3 membranes, which needed 3 and $6 \mathrm{hrs}$ for curing, respectively, were flexible. Figs. 5 are the photographs of the obtained photocured S2 and S3 membranes. The required curing time increased with decreasing PI content, as shown in Table II. The as-prepared S4 and S5 membranes were also flexible. Ethanol was removed during the photocuring. This result suggests that the PI content strongly contributed to the flexibility of the membrane.

3.2 Water uptake, ion exchange capacity, and water-resistance

Table II shows the photocuring time, water uptake, EW, IEC value, water dissolution 
temperature, and the proton conductivity at $70{ }^{\circ} \mathrm{C}$ of each sample. Although the SPEEK content was the same, water uptake and IEC values were different from each other. It is well known that SPEEK with a high SD value dissolves in water. Unfortunately, S3 and S5 membrane completely dissolved in water at 50 and $60{ }^{\circ} \mathrm{C}$, respectively. However, S2 and S4 membranes, which have higher EWs than those of S3 and S5 membrane, did not dissolve in water up to $95{ }^{\circ} \mathrm{C}$. Therefore, we could synthesize water-resistant membranes by using $2.0-5 \mathrm{wt} \%$ PI content ratios. Considering the flexibility and water-resistance of the photocured membranes in this study, $2.0-2.6 \mathrm{wt} \%$ PI content was the appropriate range for the preparation of photocured membranes to be used in a fuel cell.

\subsection{Proton conductivity}

Figure 6 shows the temperature dependence of the proton conductivity of synthesized membranes (S2, S3, S4, and S5). The data of the S1 membrane were not obtained because the membrane was too brittle and it cracked during the measurements. The proton conductivity showed the trend of S3 membrane > S5 membrane > S4 membrane $>\mathrm{S} 2$ membrane at the RH of $90 \%$. The proton conductivities of the S2, S3, S4, and S5 membranes were $19,56,28$ and $33 \mathrm{mS} \mathrm{cm}^{-1}$ at $70{ }^{\circ} \mathrm{C}$ under the $\mathrm{RH}$ of $90 \%$, respectively. 
The activation energies for the proton conduction of the S2, S3, S4, and S5 membranes were $0.24,0.25,0.22$, and $0.20 \mathrm{eV}$, respectively. These activation energies were comparable to that of Nafion ${ }^{\circledR}$ membrane. S3 membrane of the lowest PI content exhibited the highest conductivity among S2-S5 membranes, however, dissolved in water above $50{ }^{\circ} \mathrm{C}$. When the PI content was too low, the proton conductivity was found to be high, but the membrane dissolved in hot water easily.

The PI content could affect the cross-linking density directly as shown in schematic diagrams of Figs. 7(a)-(c). The double bonds of DVS monomer were consumed in the propagation process of the polymer chain. Table III shows the molar ratio of PI and other molecules. As given in the table, the average numbers of PI molecules per SPEEK polymer chain in S1 and S3 were 15 and 1.5, and the numbers of PI molecules per 1000 DVS molecules in S1 and S3 were 40 and 4.0, respectively. It should be noted that in the S3 liquid electrolyte the PI molecules were surrounded by 380 DMA molecules, which is more than four times in the S2, S4 and S5 and 10 times of S1. When the concentration of PI molecules was too low as illustrated in Fig. 7(a), some double bonds remained as a side chain group in the polymer because DVS monomer had two double bonds. When the PI content increases, these double bonds at the side chain group were activated by PI molecules and reacted with other double bonds of DVS or DVS 
derivatives. (cross-linking, Fig. 7(b)) During the cross-linking procedure, SPEEK and polymer chain derived from DVS derivatives could make semi-interpenetrating structure. As illustrated in Fig. 7(c), higher PI content may result in higher cross-linking density in the polymer. The degree of semi-interpenetrating structure could also increase with the cross-linking density. Therefore, S1, S2 and S4 membranes did not dissolve in water because they had relatively high PI content $(2.0-5.0 \mathrm{wt} \%)$. In the section 3.1 , the PI content strongly contributed to the flexibility of the membrane. When the PI content was high, the membrane became brittle because the cross-linking density was too high. This is consistent with the experimental results. It is suggested that the PI content affected the cross-linking density, which related with the water-resistance and flexibility of the membrane.

In the case of S2, S4 and S5 membranes, which contained the same amount of SPEEK, DVS, PI, and DMA as starting materials, the proton conductivity increased with the ethanol content. Although the proton conductivity of S5 membrane exhibited a high value, the membrane dissolved in water above $60{ }^{\circ} \mathrm{C}$. When the ethanol content was too large, the membrane was found to dissolve in water easily. As given in the table III, the numbers of DMA and ethanol molecules per PI molecule were 77, 170 and 260 in the S2, S4 and S5 liquid electrolytes, respectively. It means that PI molecules in the S5 
liquid electrolyte is surrounded by more than in the S2 and S4, which might be the cause of the lower cross-linking density in S5. As a result, S5 membrane dissolved in water above $60{ }^{\circ} \mathrm{C}$. These results showed that higher PI content in the liquid electrolyte might prevent the membrane to dissolve in hot water.

The membranes could have vacancies upon the removal of the solvent, such as DMA, and the vacancies worked to infiltrate more water molecules to form large proton conducting path (Fig. 4(c)). Figure 8 shows the RH dependence of the proton conductivity in each membrane. The proton conductivity increases with the RH. The proton conductivity showed the trend of S3 membrane > S5 membrane > S4 membrane > S2 membrane at the $\mathrm{RH}$ of $90 \%$. S3 membrane demonstrated the highest proton conductivity, which exhibited $18 \mathrm{mS} \mathrm{cm}^{-1}$ at the $\mathrm{RH}$ of $90 \%$ and $25{ }^{\circ} \mathrm{C}$. The initial content of PI and ethanol in the liquid precursor was found to strongly affect the proton conductivity and water-resistance, even though obtained membrane did not contain them anymore.

3.4 Demonstration of the proton-conductive patterned membrane

Figures 9(a) and 9(b) show the scanning electron micrograph and photograph of the patterned structure on Si wafer and patterned membrane, respectively. The dimension of 
the square features was $\sim 3 \times 3 \mu \mathrm{m}$ and periodicity was $\sim 6 \mu \mathrm{m}$. The patterned membrane had the negative image of the silicon master. A fine micro-structure was successfully obtained on the surface of the photocured membrane.

\section{Conclusion}

Photocurable electrolytes based on proton conductive polymer, sulfonated poly(ether ether ketone) (SPEEK), were prepared to investigate the proton transport property. Water uptake, ion exchange capacity, proton conductivity, and water-resistance were investigated for a series of photocurable electrolytes with various PI and solvent ratios. It was demonstrated that the water-resistant photocured membrane is easy to prepare, which gives relatively high proton conductivity $\left(28 \mathrm{mS} \mathrm{cm}{ }^{-1}\right.$ at $70{ }^{\circ} \mathrm{C}$ and relative humidity of $90 \%)$. The initial PI content in the liquid precursor was found to strongly affect the proton conductivity, water-resistance, and flexibility. The optimum PI content was $2.0-2.6 \mathrm{wt} \%$ for the preparation of the photocured membrane in this work. A patterned micro-structure on the photocured membrane was also demonstrated by using the property of the photocurable electrolytes.

\section{Acknowledgments}


We gratefully thank Dr. Isao Aritome (JSR Corporation, Japan) for valuable advice. This study was supported by Improvement program of Research Environment for Young Researchers from Special Coordination Funds for Promoting Science and Technology (SCF) commissioned by the Ministry of Education, Culture, Sports, Science and Technology (MEXT) of Japan. This work was partly supported by the Iketani Science and Technology Foundation, Hattori-Hokokai, JST PRESTO program, and the Japan Society for the Promotion of Science (JSPS) through the Funding Program for Next Generation World-Leading Researchers (NEXT Program), initiated by the Council for Science and Technology Policy (CSTP). 


\section{References}

1) J. Schmeisser, S. Holdcroft, J. Yu, T. Ngo, and G. McLean, Chem. Mater. 17 (2005) 387.

2) L. Depre, J. Kappel, and M. Popall, Electrochim. Acta 43 (1998) 1301.

3) Z. Zhou, R. N. Dominey, J. P. Rolland, B. W. Maynor, A. A. Pandya, and J. M. DeSimone, J. Am. Chem. Soc. 128 (2006) 12963.

4) L. Chikh, V. Delhorbe, and O. Fichet, J. Membr. Sci. 368 (2011) 1.

5) C. A. Dai, C. P. Liu, Y. H. Lee, C. J. Chang, C. Y. Chao, and Y. Y. Cheng, J. Power Sources 177 (2008) 262.

6) T. Kobayashi, M. Rikukawa, K. Sanui, and N. Ogata, Solid States Ionics 106 (1998) 219.

7) R. Y. M. Huang, P. Shao, C. M. Burns, and X. Feng, J. Appl. Polym. Sci. 82 (2001) 2651.

8) P. Xing, G. P. Robertson, M. D. Guiver, S. D. Mikhailenko, K. Wang, and S. Kaliaguine, J. Membr. Sci. 229 (2004) 95.

9) S. M. J. Zaidi, S. D. Mikhailenko, G. P. Robertson, M. D. Guiver, S. Kaliaguine, J. Membr. Sci. 173 (2000) 17.

10) T. A. Zawodzinski Jr., M. Neeman, L. O. Sillerud, and S. Gottesfeld, J. Phys. Chem. 
95 (1991) 6040. 


\section{Figure captions}

Fig. 1 Chemical structures of the materials.

Fig. $2{ }^{1} \mathrm{H}$ NMR spectrum of the synthesized SPEEK in DMSO-d6.

Figs. $3{ }^{1} \mathrm{H}$ NMR spectra of the photocured membranes before and after heat treatment.
(a) $\mathrm{S} 2$ membrane
(b) S3 membrane.

Figs. 4 Schematic illustration of the state of photocured membrane by heat treatment and hydration. SPEEK was embedded in the photocured membrane derived from the polymerized reaction of DVS. (a)Solvent such as DMA still remained in the photocured membrane. Some of the DMA molecules made hydrogen bonds with the protons of $-\mathrm{SO}_{3} \mathrm{H}$ groups., (b)Solvent was removed from the photocured membrane by heat treatment, (c)Water infiltrated into the vacancy in the photocured membrane. Large proton conduction path was formed.

Figs. 5 Photographs of synthesized membranes. (a) S2 membrane and (b) S3 membrane.

Fig. 6 Temperature dependence of the proton conductivity. $\boldsymbol{\Delta}:$ S2 membrane, $\bullet$ S3

membrane, $\mathbf{~ : ~ S 4 ~ m e m b r a n e , ~} \bullet$ : S5 membrane.

Figs. 7 Schematic illustration for increasing the cross-linking density with the PI content. (a)Propagation without cross-linking (low PI content), (b)Low cross-linking density with medium PI content, (c)High cross-linking density with high PI 
content and interpenetrating structure for SPEEK.

Fig. 8 Relative humidity dependence of the proton conductivity. $\mathbf{\Delta}$ : S2 membrane, S3 membrane, $\square:$ S4 membrane, $\bullet:$ S5 membrane.

Figs. 9 (a) Scanning electron micrograph of the patterned Si wafer. (b) Photograph of the patterned membrane observed by optical microscope. 


\section{Table captions}

Table I. Compositions of liquid photocurable electrolytes (unit : g).

Table II. Effect of PI content on properties of photocured membranes. ${ }^{a}$ The values were obtained at room temperature. ${ }^{\mathrm{b}}$ Measurements were carried out at $70{ }^{\circ} \mathrm{C}$.

Table III. Molar ratio of PI and other molecules. ${ }^{\text {a }}$ Number of PI molecules per one polymer chain of SPEEK. ${ }^{b}$ Number of PI molecules per 1000 molecules of DVS. ${ }^{\mathrm{c}}$ Number of DMA molecules per PI molecule. ${ }^{\mathrm{d}}$ Number of ethanol molecules per PI molecule. ${ }^{c}$ Number of DMA and ethanol molecules per PI molecule. 
Table I.

\begin{tabular}{cccccc}
\hline Sample & SPEEK & DVS & PI & DMA & Ethanol \\
\hline S1 & $0.8 \pm 0.01$ & $1.4 \pm 0.01$ & $0.2 \pm 0.01$ & $1.6 \pm 0.01$ & 0 \\
S2 & 0.8 & 1.4 & 0.1 & 1.6 & 0 \\
S3 & 0.8 & 1.4 & 0.02 & 1.6 & 0 \\
S4 & 0.8 & 1.4 & 0.1 & 1.6 & $1.0 \pm 0.1$ \\
S5 & 0.8 & 1.4 & 0.1 & 1.6 & 2.0 \\
\hline
\end{tabular}


Table II.

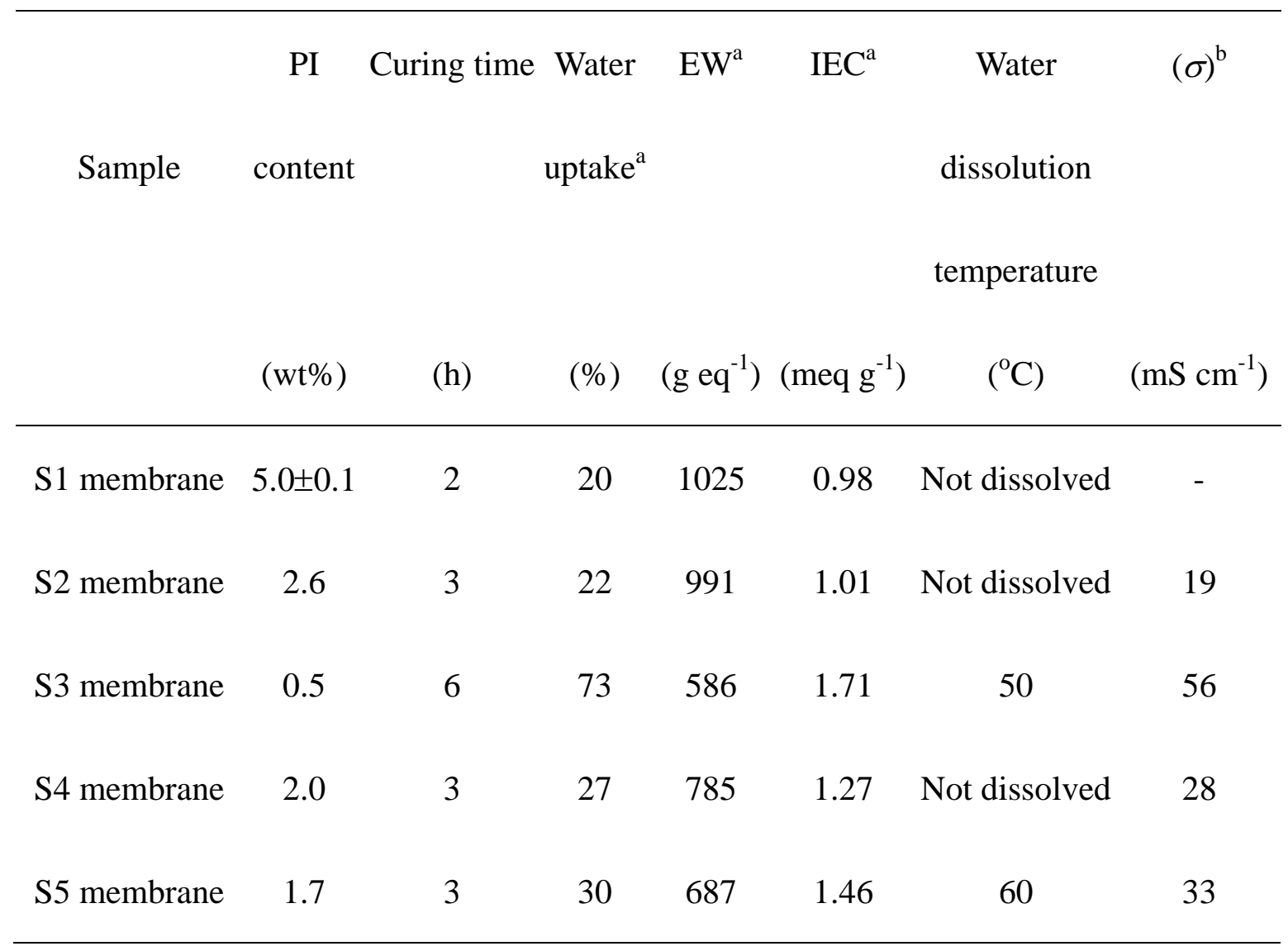


Table III.

\begin{tabular}{|c|c|c|c|c|c|}
\hline Sample & $\mathrm{PI} / \mathrm{SPE}$ & 1000 & MA / & anol / & Ethanol) / $\mathrm{PI}^{\mathrm{e}}$ \\
\hline $\mathrm{S} 1$ & 15 & 40 & 38 & 0 & 38 \\
\hline $\mathrm{S} 2$ & 7.6 & 20 & 77 & 0 & 77 \\
\hline $\mathrm{S} 3$ & 1.5 & 4.0 & 380 & 0 & 380 \\
\hline $\mathrm{S} 4$ & 7.6 & 20 & 77 & 91 & 170 \\
\hline S5 & 7.6 & 20 & 77 & 180 & 260 \\
\hline
\end{tabular}




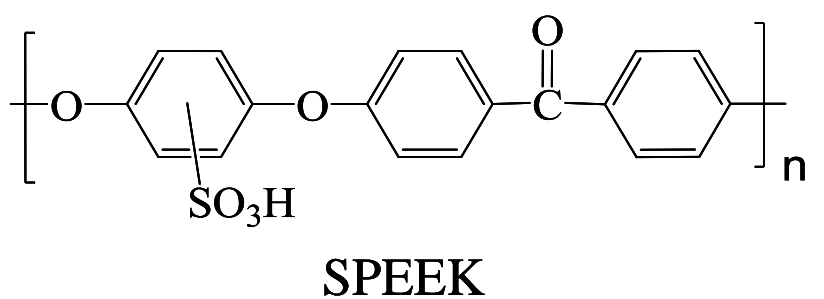

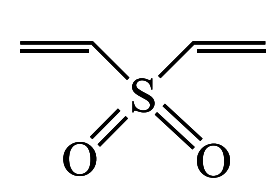

DVS

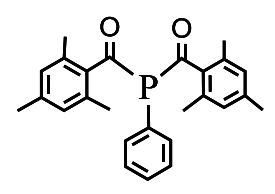

PI

Fig. 1 

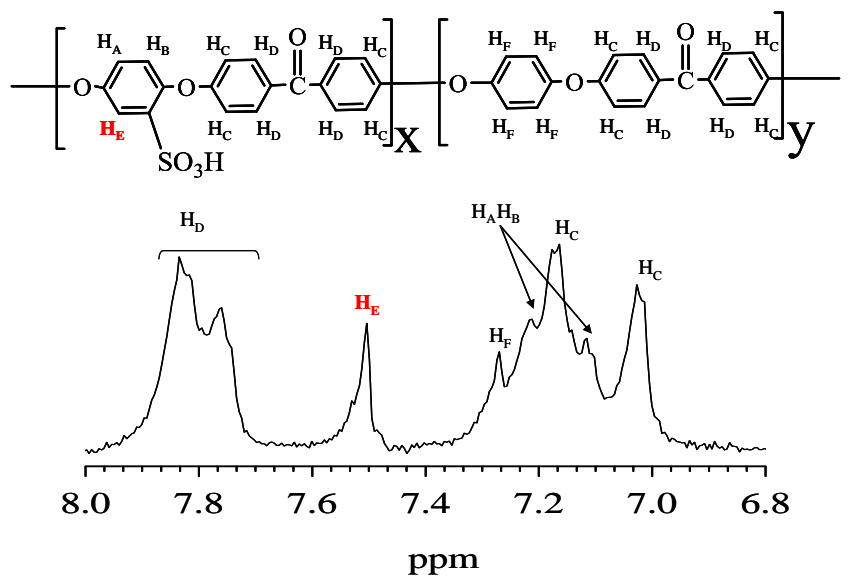

Fig. 2 
(a)

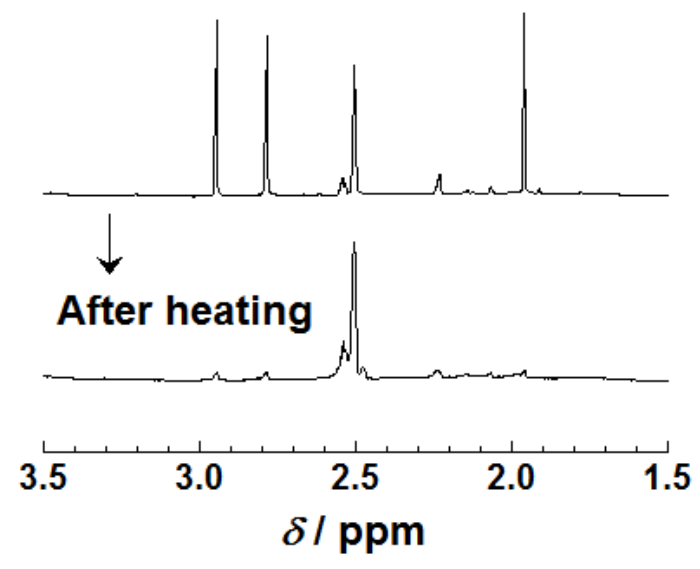

(b)

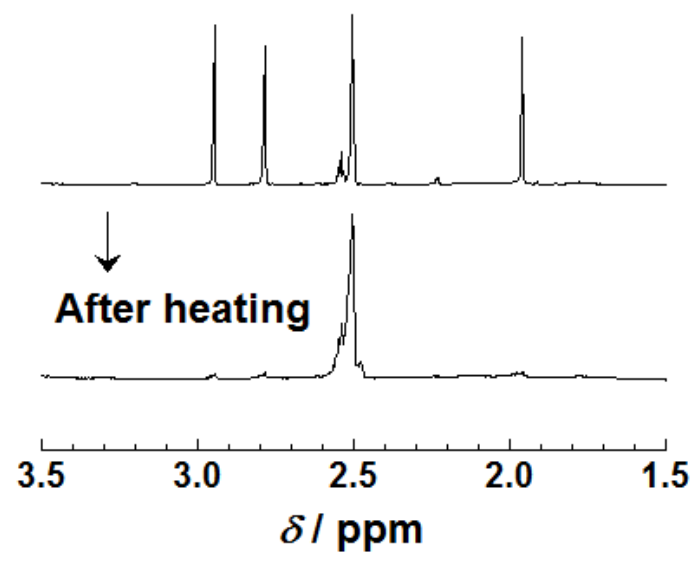

Figs. 3 
(a)

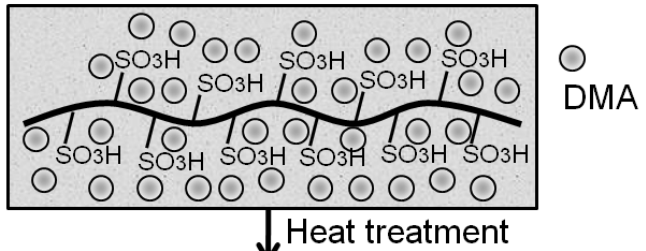

(b)

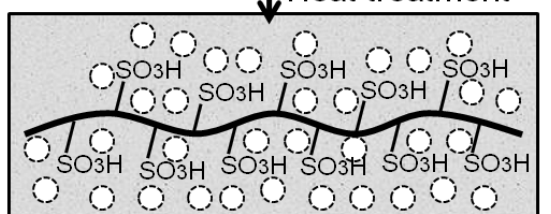

$\downarrow$ Hydration

(c)

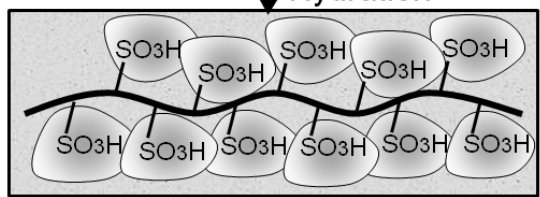

Figs. 4 

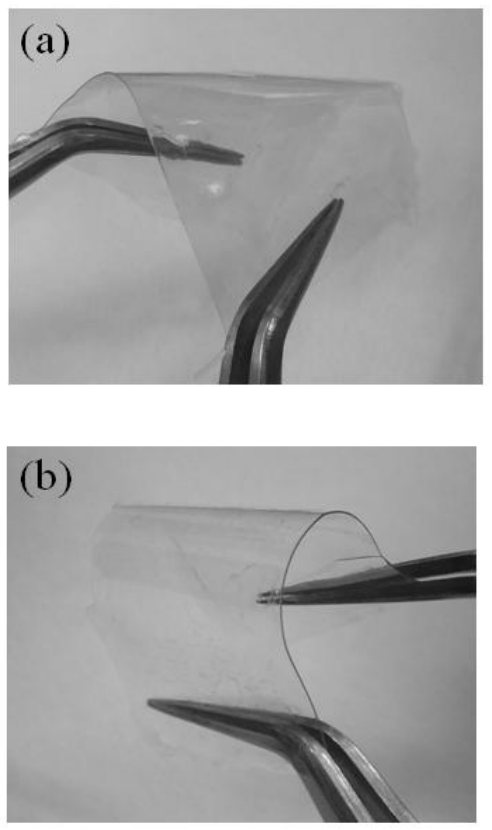

Figs. 5 


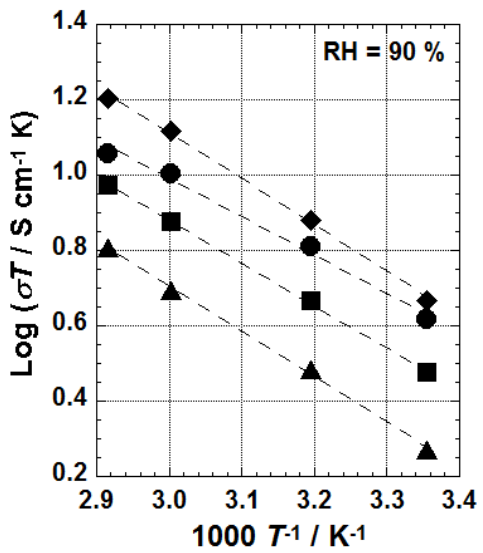

Fig. 6 
(a)

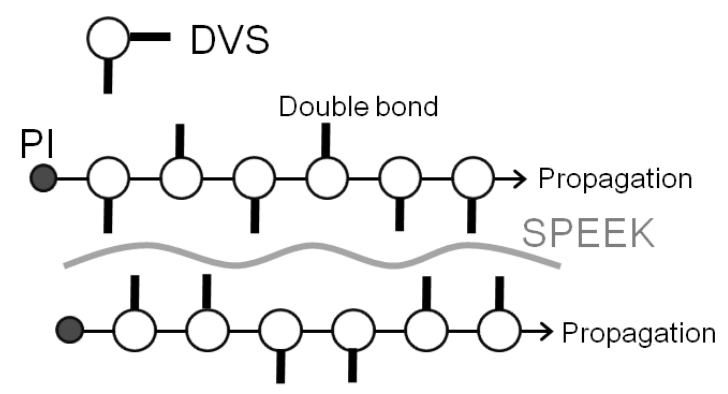

(b)

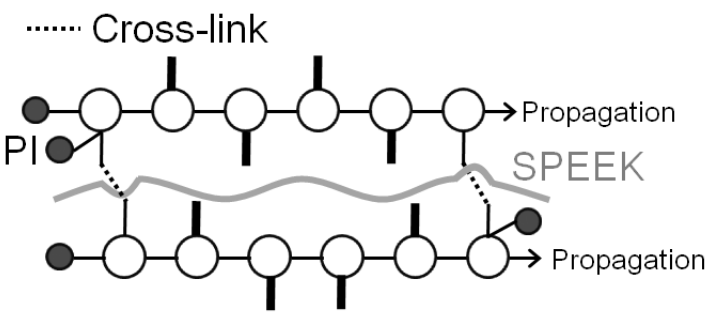

(c)

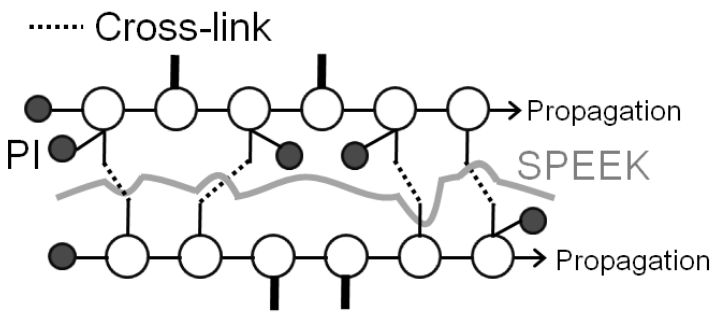

Figs. 7 


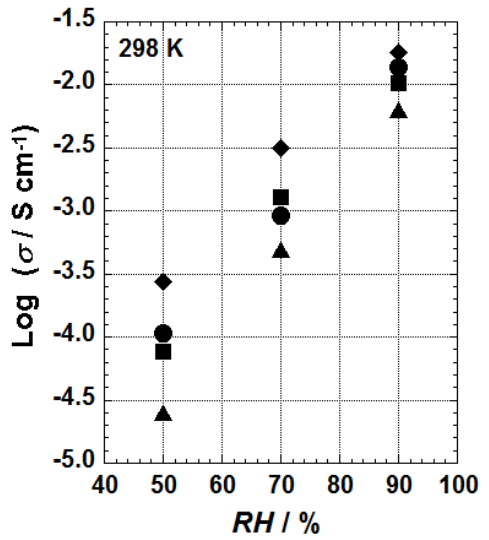

Fig. 8 
(a)

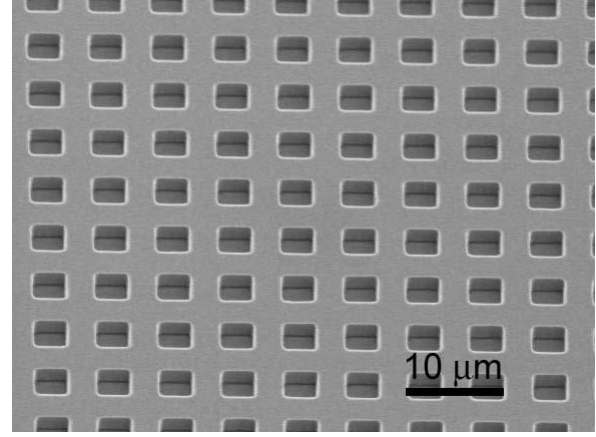

(b)

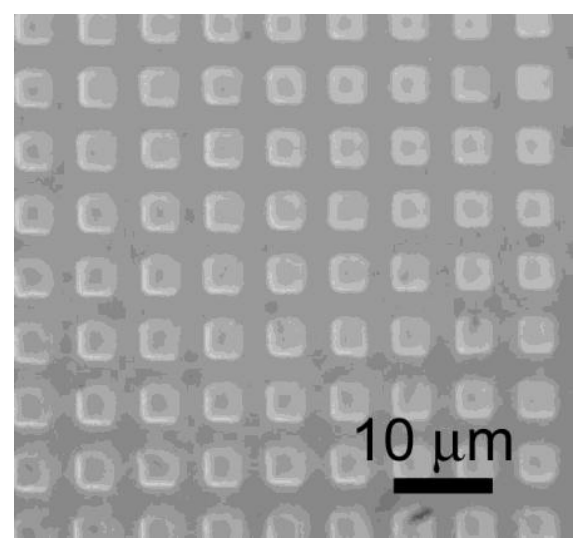

Figs. 9 